COMUNICAÇÃO CIENTÍFICA

\title{
FIXAÇÃO DE FRUTOS DE LIMEIRAS ÁCIDAS \\ 'TAHITI' ANELADAS E TRATADAS COM ÁCIDO GIBERÉLICO ${ }^{1}$
}

\author{
CASSIANO SPAZIANI PEREIRA², DALMO LOPES DE SIQUEIRA ${ }^{3}$, \\ LUIZ CARLOS CHAMUM SALOMÃO ${ }^{4}$, PAULO ROBERTO CECON ${ }^{5}$
}

RESUMO-O objetivo deste trabalho foi avaliar o efeito do ácido giberélico $\left(\mathrm{GA}_{3}\right)$ e do anelamento de ramos sobre a abscisão de estruturas florais e o pegamento de frutos em limeira ácida 'Tahiti'. Para as variáveis relacionadas à abscisão de estruturas reprodutivas, o esquema experimental foi em parcelas subdivididas no tempo, com o fatorial $4 \times 3$ nas parcelas. Quanto ao pegamento de frutos, o esquema experimental foi apenas o fatorial 4 x 3 . O primeiro fator foi a aplicação de $\mathrm{GA}_{3}$ em quatro concentrações $(0 ; 7 ; 14 \mathrm{e} 21 \mathrm{mg} / \mathrm{L})$, e o segundo, as duas épocas de anelamento: no início do florescimento (AIF) e a um mês após o florescimento (AMAF), mais a testemunha sem anelamento (SA). O delineamento experimental foi em blocos casualizados (DBC), com cinco repetições. Verificou-se que o $\mathrm{GA}_{3}$ não inibe a abscisão das estruturas reprodutivas e não afeta o pegamento dos frutos de limeira ácida 'Tahiti'. O anelamento reduziu a abscisão das estruturas reprodutivas. O pegamento de frutos nas plantas com o AIF foi 15,66\% e com o AMAF, de 16,11\%, o que significa aumentos de $220 \%$ e $229 \%$, respectivamente, em relação às plantas SA, que tiveram pegamento de $4,89 \%$. Termos para indexação: anelamento de ramos, ácido giberélico, limeira ácida 'Tahiti”, pegamento de frutos, abscisão de frutos.

\section{FLORAL STRUCTURES AND FRUIT SETTING OF ACID LIME TREES 'TAHITI' GIRDLED AND TREATED WITH GIBBERELLIC ACID}

\begin{abstract}
The objective of this work was to evaluate the effect of the gibberellic acid and of the girdling of branches on the abscission of floral structures and on the fruit setting of acid lime tree 'Tahiti'. For the variables related to the abscission of the reproductive structures, the experimental design was in split plots in time, with the factorial $4 \times 3$ in the plots. As to the fruit setting, the experimental design was the factorial $4 \times 3$. The first factor was the application of $\mathrm{GA}_{3}$ in four concentrations $(0,7,14$ and $21 \mathrm{mg} / \mathrm{L})$, and the second one, the two periods of girdling: one at the beginning of flowering (AIF) and the other one a month later (AMAF), plus the control without girdling (SA). The experimental design was in randomized blocks (DBC), with five repetitions. It was observed that the $\mathrm{GA}_{3}$ did not change the abscission of the reproductive structures and neither the fruit setting of the acid lime tree 'Tahiti'. The girdled reduced the abscission of the reproductive structures. The setting in the AIF plants was of $15.66 \%$, and, in the AMAF plants of $16.11 \%$, which means an increasing of 220 and $229 \%$, respectively, in relation to the SA plants that had a setting of $4.89 \%$.
\end{abstract}

Index terms: branch girdling, gibberellic acid, "Tahiti” lime fruit, fruit set, fruit abscission.

\footnotetext{
${ }^{1}$ (Trabalho 240-09). Recebido em: 19-10-2009. Aceito para publicação em: 27-05-2010.

${ }^{2}$ Eng. Agr. DSc, Prof. Adjunto da Universidade Federal do Mato Grosso, email: caspaziani@yahoo.com.br

${ }^{3}$ Eng. Agr. DSc Prof. Associado da Universidade Federal de Viçosa, Dept ${ }^{\circ}$. de Fitotecnia, Viçosa - MG, Siqueira@ufv.br

${ }^{4}$ Eng. Agr. DSc Prof. Associado da Universidade Federal de Viçosa, Dept ${ }^{\circ}$. de Fitotecnia, Viçosa - MG, Luiz.salomão@pq.cnpq.br

${ }^{5}$ Eng. Agr.,DSc, Professor Associado da Universidade Federal de Viçosa, Dept ${ }^{\circ}$. de informática, Viçosa-MG, email: paulo.cecon@pq.cnpq.br
} 
Durante a floração dos Citrus, ocorre forte competição por carboidratos entre flores, frutos e o crescimento vegetativo. Quando o florescimento é muito intenso, os teores de carboidratos nos tecidos diminuem, havendo um comando interno, possivelmente hormonal, que aciona o processo de abscisão, equilibrando os teores de carboidratos com o número de frutos que irão chegar à colheita (RIVAS et al., 2007).

A abscisão de flores e frutos em limeira ácida 'Tahiti' é mais acentuada do que nas cultivares com sementes. As principais causas da abscisão em citros são os desequilíbrios hormonais e a intensa floração em cachos florais desprovidos de folhas maduras (fontes) (SCHAFER et al., 1999).

No processo de abscisão, estão envolvidos o ácido abscísico, o etileno e as giberelinas. O etileno e o ácido abscísico promovem a abscisão, e as giberelinas reduzem a abscisão por meio do aumento da divisão e alongamento celular, aumentando a força de dreno do órgão (TALÓN et al., 1998).

Com o intuito de reduzir a abscisão, recomenda-se a aplicação de ácido giberélico $\left(\mathrm{GA}_{3}\right)$, que estimula a formação de folhas novas e aumenta a área foliar, incrementando, consequentemente, a síntese de fotoassimilados, o crescimento de ramos e o pegamento de frutos (SHANI et al., 2006).

Outra prática recomendada para aumentar o pegamento de frutos é o anelamento de ramos. $\mathrm{O}$ anelamento consiste na confecção de dois cortes paralelos e posterior retirada de uma pequena porção da casca, em toda a circunferência do tronco (SATORI; ILHA, 2005).

$\mathrm{O}$ anelamento promove retenção temporária de carboidratos na parte aérea das plantas, impedindo a descida dos fotoassimilados até as raízes, disponibilizando com isso mais carboidratos para os frutos e aumentando, consequentemente, o pegamento (RIVAS et al., 2006; RIVAS et al., 2007).

Diante do exposto, o objetivo deste trabalho foi avaliar o efeito do anelamento e da aplicação de $\mathrm{GA}_{3}$ sobre o pegamento de frutos em limeira ácida 'Tahiti'.

O experimento foi conduzido no Pomar do "Fundão", do Setor de Fruticultura da Universidade Federal de Viçosa (UFV) - Viçosa-MG, no período de 18-08-2006, início do florescimento, até o final da colheita dos frutos, no final de abril de 2007.

Para o experimento, foram utilizadas plantas de limeira ácida 'Tahiti' (Citrus latifolia Tanaka), em campo, com aproximadamente oito anos de idade, enxertadas sobre o limoeiro 'Cravo' (Citrus limonia Osbeck). As copas das plantas ainda estavam em crescimento devido a uma poda drástica realizada em
2005. O espaçamento utilizado foi de 7 x 3 metros. A adubação seguiu as recomendações da Comissão de Fertilidade do Solo do Estado de Minas Gerais (1999). Durante o experimento, as plantas não foram irrigadas.

O esquema experimental utilizado para as variáveis de abscisão de estruturas reprodutivas foi em parcelas subdivididas no tempo, com o fatorial 4 × 3 nas parcelas. Para o pegamento de frutos, foi empregado o esquema fatorial $4 \times 3$. O primeiro fator consistiu na aplicação de $\mathrm{GA}_{3}$ em quatro concentrações (0; 7; 14 e 21 mg/L), em 13-9-2006. O segundo fator foram as duas épocas de anelamento: Anelamento no início do florescimento (AIF), realizado em 21-8-2006, e anelamento um mês após o florescimento (AMAF), realizado em 21-9-2006, mais a testemunha sem anelamento (SA). O delineamento adotado foi em blocos casualizados, com cinco repetições e duas plantas por parcela.

As caldas com $\mathrm{GA}_{3}$ foram aplicadas dia 1309-2006, constituídas de $\mathrm{GA}_{3}$, água e espalhante adesivo siliconado, não sendo controlado o $\mathrm{pH}$ da calda. A aplicação do $\mathrm{GA}_{3}$ nas plantas foi com auxílio de um pulverizador manual, quando cerca de $80 \%$ das pétalas haviam sofrido abscisão, seguindo recomendações de Primo-Millo (1993).

Os anelamentos foram realizados a aproximadamente $15 \mathrm{~cm}$ acima do local de enxertia, rompendo-se o floema em toda a sua circunferência, removendo porções da casca de $5 \mathrm{~mm}$ de espessura. Tomou-se o cuidado de não aprofundar demasiadamente o corte para evitar danos ao xilema, o que promove extravasamento de seiva, interrompendo temporariamente o fluxo ascendente para região acima do anelamento (SARTORI et al., 2003).

Foram avaliados a abscisão de estruturas reprodutivas, o pegamento e a produção de frutos.

Para determinar a abscisão de estruturas reprodutivas, foram colocadas redes do tipo "clarite" sob a copa das plantas, e a cada 15 dias, as estruturas reprodutivas foram coletadas, contadas e classificadas em botões florais, flores e frutos.

A colheita foi do final de fevereiro até o final de abril, quando os frutos apresentavam a coloração verde-clara e casca lisa, características de frutos com teor de suco superior a 40\% (GAYET et al.,1995).

Os dados obtidos foram submetidos à análise de variância e de regressão. As médias foram comparadas, utilizando-se do teste de Tukey, a 5\% de probabilidade. As análises estatísticas foram realizadas com o auxílio do software "Sisvar" (FERREIRA, 2000).

$\mathrm{O} \mathrm{GA}_{3}$ não teve efeito sobre a abscisão das estruturas reprodutivas, número de frutos colhidos 
e o pegamento de frutos de limeira ácida 'Tahiti'.

A ausência de efeitos do $\mathrm{GA}_{3}$ pode ter ocorrido devido às concentrações de carboidratos nos tecidos das plantas, no momento da aplicação (TALÓN et al., 1998); estado nutricional das plantas; época de aplicação; concentração de $\mathrm{GA}_{3}$ aplicada; condições ambientais (GUARDIOLA, 1996), natureza do espalhante (GUARDIOLA, 1988) e pH da calda, que não foi monitorado, sendo o recomendado $\mathrm{pH} 4,5$ (CASAGRANDE JR. et al., 1999).

Verificou-se que a abscisão de botões florais foi maior nas plantas SA e AMAF, 10,7 e 12,1 botões/ parcela, respectivamente. A menor abscisão ocorreu nas plantas com AIF (4,15 botões/parcela), redução de 57,94\% em relação às plantas SA (Figura 1a).

A abscisão de flores nas plantas SA e com AMAF foi 93,1 e 94,85 flores/parcela, respectivamente. Nas plantas com AIF, a abscisão foi de 31,8 flores/ parcela, (redução de $65,84 \%$ em relação às plantas SA) (Figura 1b). A abscisão de frutos não teve diferença entre os tratamentos (Figura 1c).
As duas épocas de anelamento aumentaram o pegamento de frutos, não havendo diferença significativa entre elas. As plantas com o AIF e o AMAF tiveram pegamento de 15,66 e $16,11 \%$, aumento de 220 e $229 \%$, respectivamente, em comparação com as plantas SA, que tiveram pegamento de $4,89 \%$ (Figura 2).

O aumento do pegamento de frutos ocorreu devido à capacidade do anelamento em reduzir a abscisão de estruturas florais, isso porque o anelamento bloqueia o transporte via floema de fotoassimilados, hormônios e nutrientes, da parte aérea das plantas para as raízes, promovendo um acúmulo destas substâncias na parte aérea das plantas (RIVAS et al., 2006; TAKAYOSHI, 2006; RIVA et al.,2007; MEHOUACHI et al., 2009).

$\mathrm{O} \mathrm{GA}_{3}$ não contribui para aumentar o pegamento de frutos. $\mathrm{O}$ anelamento do início do florescimento até um mês depois contribui para aumentar o pegamento de frutos.

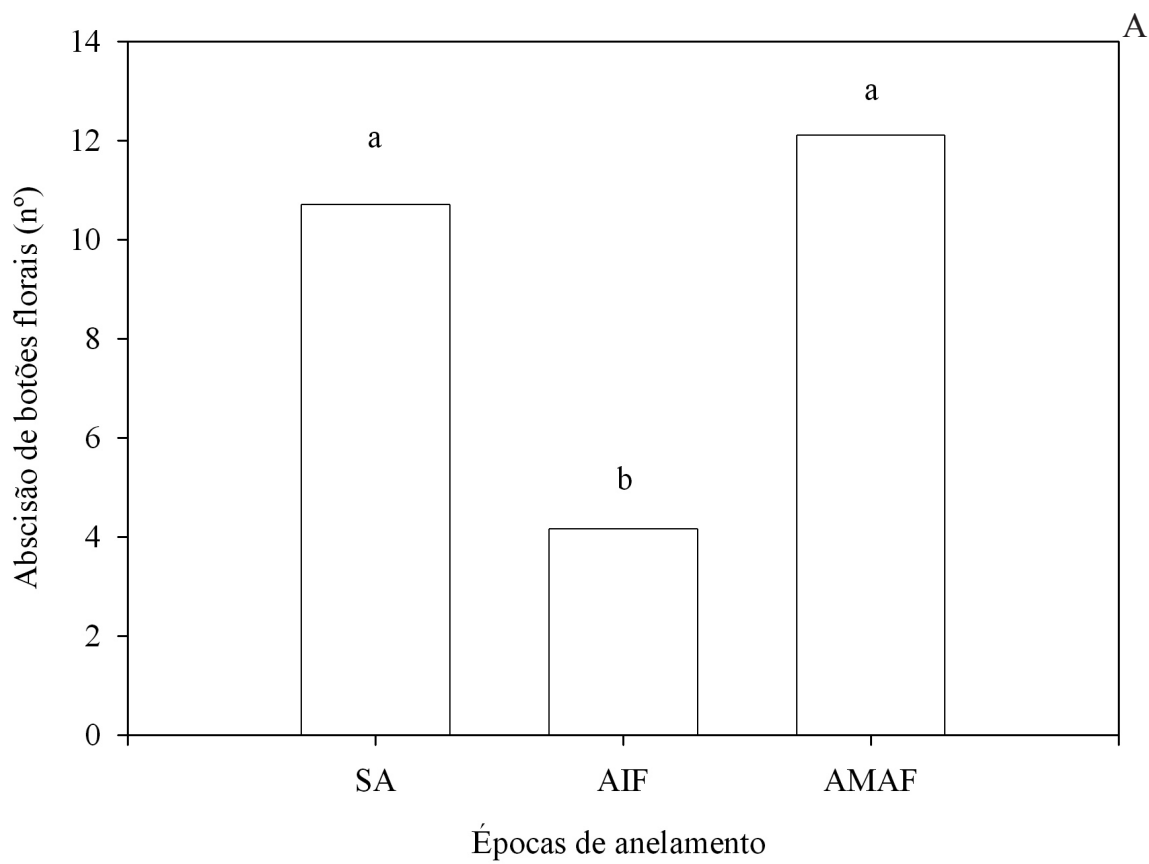




\section{continuação}


$\mathrm{C}$

FIGURA 1 -Abscisão acumulada de botões florais (a), flores (b) e frutos (c) em plantas de limeira ácida 'Tahiti', nas três épocas de anelamento: sem anelamento (SA), anelamento no início do florescimento (AIF) e anelamento um mês após o florescimento (AMAF). Médias seguidas das mesmas letras não diferem entre si, a 5\% de probabilidade, pelo teste de Tukey. 


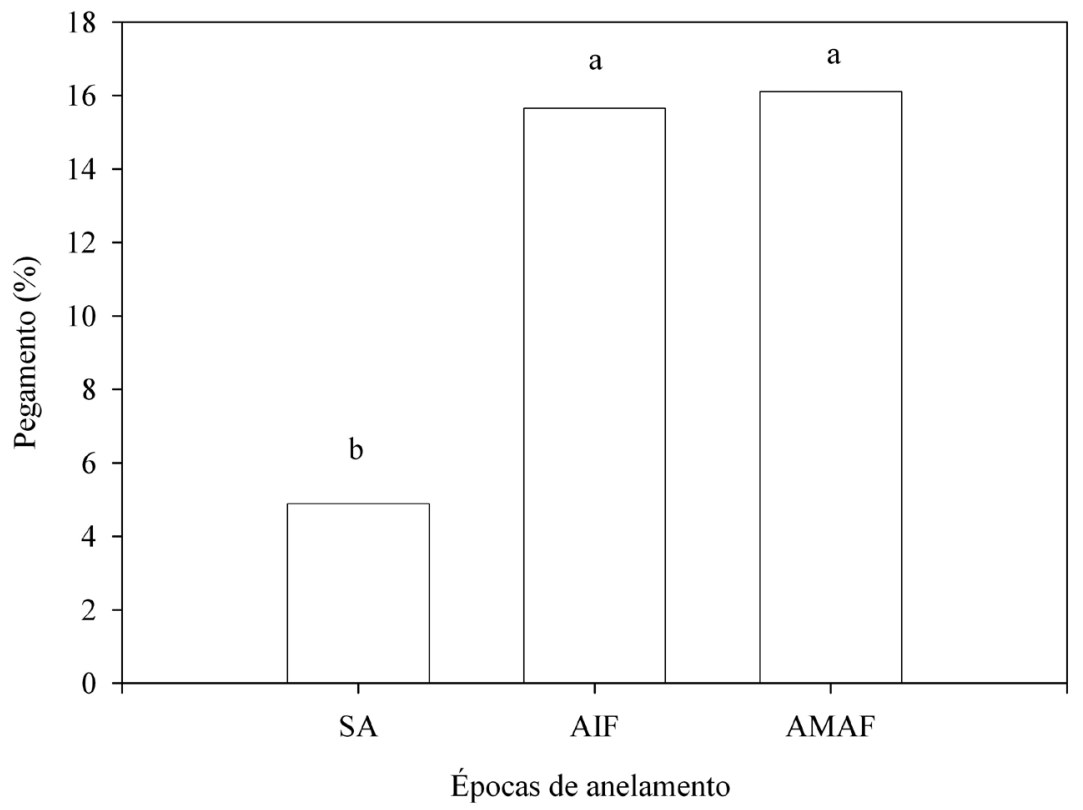

FIGURA 2 - Pegamento de frutos de limeira ácida 'Tahiti', na florada de setembro de 2006, nos tratamentos sem anelamento (SA), anelamento no início do florescimento (AIF) e anelamento um mês após o florescimento (AMAF). Médias seguidas das mesmas letras não diferem entre si, a 5\% de probabilidade, pelo teste de Tukey.

\section{REFERÊNCIAS}

CASAGRANDE JR., J. G.; FACHINELLO, J. C.; FARIA, J. L. C. O pH da calda de aplicação e a absorção de ácido giberélico por frutas de laranja cv. 'Valência'. Scientia Agrícola. v.56 n.4, p. 9339381999.

COMISSÃO DE FERTILIDADE DO SOLO DO ESTADO DE MINAS GERAIS. Recomendações para o uso de corretivos e fertilizantes em Minas Gerais: 5a Aproximação. Viçosa-MG, 1999. 359 p.

FERREIRA, D.F. Sistema de análises de variância para dados balanceados. Lavras: UFLA, 2000. (SISVAR 4. 1).

GAYET, J.P.; BLEINROTH, E.W.; MATALLO, M.; GARCIA, E.E.C.; GARCIA, A.E.; ARDITO, E.F.G.; BORDIN, M.R. Lima ácida 'Tahiti' para a exportação: procedimentos de colheita e póscolheita. Brasília: EMBRAPA - SPI, 1995. 36 p. (Série Publicações Técnicas FRUPEX, 12).

GUARDIOLA, J. L. Factor limiting productivity in citrus: a physiological approach. In: INTERNATIONAL CITRUS SYMPOSIUM, 6., 1998. Proceedings... Riverside: Universidade of California, 1988.
GUARDIOLA, J. L. Future use of plant bioregulators. In: INTERNATIONAL CITRUS CONGRESS, 8. 1996. Proceedings... Sun City: International Society of Citriculture, 1996. v. 2, p. 938-944.

MEHOUACHI, J.; IGLESIAS, D. J.; AGUSTÍ, M.; TALÓN, M. Delay of early fruitlet abscisión by Branco girdling in Citrus coincides with previous increases in carbohydrate and gibberelin concentrations. Plant Growth Regulator. Farnham Royal, v.58, p 15-23, 2009.

PRIMO-MILLO, E. Regulacion del cuajado del fruto en los citricos. In: CONGRESSO DE CITRICULTURA DE LA PLANA, 1., 1993. Anais... Valência: Ajunta de Nules, 1993. p.57-74.

RIVAS, F.; ERNER, E.; ALÓS, E.; JUAN, M.; ALMELA, V.; AGUSTÍ, M. Girdling increases carbohydrate availability and fruit-set in citrus cultivars irrespective of parthenocarpic ability. Journal of Horticultural Science \& Biotechnology, Ashford, v. 81, n. 2 , p. $289-295,2006$.

RIVAS, F.; GRAVINA, A.; AGUSTÍ, M. Girdling effects on fruit set and quantum yield efficiency of PSII in two Citrus cultivars. Tree Physiology, Victoria, v.27, p. 527-535, 2007. 
SARTORI, I. A. Guerra, D.S.; Marodin, G.A.B. . Aplicação de auxinas e incisão anelar em pessegueiros da cv. Sentinela. Ciência Rural, Santa Maria, v. 33 , n. 2, p. 247-253, 2003.

SARTORI, I. A.; ILHA, L. L. H. Anelamento e incisão anelar em fruteiras de caroço. Ciência Rural, Santa Maria, v.35, n.3, p. 724-729, 2005.

SCHAFER, G.; KOLLER, O. C.; SARTORI, I. A. Retenção de frutos de laranjeiras-de-umbigo 'Monte Parnaso' em função da aplicação de 2,4 D, ácido giberélico, e da anelagem de ramos. Ciência Rural, Santa Maria,v. 29, n. 4, p.639-644, 1999.

SHANI, E.; YANAI, O.; ORI, N. The role of hormonees in shoot apical meristem function. Current Opinion in Plant Biology, London, .9, p.484-489, 2006.
TAKAYOSHI, Y. Effects of trunk girdling and crop load levels on fruit quality and root elongation in 'Aki Queen' grapevines. Journal of the Japanese Society for Horticultural Science, Tokyo, v.75, p.439-444, 2006.

TALÓN, M.; TADEO, F. R.; BEN-CHEIKH, W.; GOMES-CADENAS, A.; MEHOUACHI, J.; PÉREZ-BOTELLA, J.; PRIMO-MILLO, E. Hormonal regulation of fruit set and abscission in citrus: classical concepts and new evidence. Acta Horticulturae, Wageningen, v.463, p.209-218, 1998. 